\title{
Dichlorvos Induced Autoimmune Hepatitis: A Case Report and Review of Literature
}

\author{
Su Xian Zhao ${ }^{1}$; Qing Shan Zhang ${ }^{1}$; Li Kong ${ }^{1, *}$; Yu Guo Zhang ${ }^{1}$; Rong Qi Wang ${ }^{1}$; Yue Min Nan ${ }^{1}$; \\ Ling Bo Kong ${ }^{1}$ \\ ${ }^{1}$ Department of Traditional and Western Medical Hepatology, Third Hospital of Hebei Medical University, Shijiazhuang, China \\ *Corresponding Author: Li Kong, Department of Traditional and Western Medical Hepatology, Third Hospital of Hebei Medical University, Shijiazhuang, China. Tel: +86-31166781228, \\ Fax:+86-31187023626, E-mail: kongliyouxiang@sina.com
}

Received: November 19, 2014; Revised: January 31, 2015; Accepted: February 13, 2015

\begin{abstract}
Introduction: Drug-induced liver injury is a frequent cause of hepatic dysfunction. Several drugs have been identified to cause autoimmune hepatitis (AIH). Environmental chemicals are capable of triggering a certain degree of liver injury. However, toxin induced AlH is rare.

Case Presentation:We reported a woman with chronic (long-term) exposures to dichlorvos, which resulted in liver injury and cirrhosis. She was diagnosed after a second liver biopsy, which was correlated with laboratory findings. At the same time, she experienced hepatic cortical blindness during her first admission.

Conclusions: Chronic (long-term) exposures to dichlorvos can lead to AIH. A detailed inquiry of medical history and liver biopsy are necessary for the diagnosis of toxin-induced AIH. Corticosteroid therapy is associated with favorable evolution.
\end{abstract}

Keywords: Dichlorvos; Autoimmune Hepatitis; Drug-Induced Liver Injury

\section{Introduction}

Autoimmune hepatitis (AIH) is a chronic necroinflammatory liver disease of unknown etiology with yearly increases in incidence. The diagnosis of the disease is based on the clinical and laboratory findings, as well as the exclusion of other causes of chronic liver diseases, such as drug-induced liver injury (DILI), viral hepatitis, Wilson disease and overlap syndrome (1-5). Recently, a growing number of drug-induced autoimmune liver diseases (DIAILD) were reported. The DILI is a potential complication that can occur with the intake of many medications, herbs and/or dietary supplements. Multiple drugs and environmental chemicals are capable of evoking a certain degree of liver injury. Several drugs have been identified to cause AIH that may persist after discontinuation, suggesting that they triggered true autoimmunity. These include oxyphenisatin, nitrofurantoin, minocycline, chlometacin and alpha-methyl dopa $(6,7)$. Recently, drug-induced autoimmune hepatitis (DIAIH) has been reported to be caused by several drugs, while toxin agent induced AIH is rare (8). We reported here a woman with chronic (long-term) exposures to dichlorvos, which resulted in liver injury and cirrhosis. She was diagnosed with AIH after a second liver biopsy, correlated with laboratory features. At the same time, she has experienced hepatic cortical blindness during her first admission.

\section{Case Presentation}

A 49-year-old Chinese woman was admitted to our hospital for the second time with complaints of loss of appetite, tiredness, feeling weak, intermittent dark colored urine and abnormal liver function for two and a half years. Two and half years prior to admission, the patient presented for the same symptoms and also jaundice. Initially, she presented the following values for serum parameters: alanine transaminase (ALT) 1558 $\mathrm{U} / \mathrm{L}$ (normal $5-40 \mathrm{U} / \mathrm{L}$ ), aspartate transaminase (AST) $1267 \mathrm{U} / \mathrm{L}$ (normal 10 - $40 \mathrm{U} / \mathrm{L}$ ), total bilirubin (TBIL) 133.5 umol/L (normal 3 - $20 \mathrm{umol} / \mathrm{L}$ ), alkaline phosphatase (AKP) $182 \mathrm{U} / \mathrm{L}$ (normal 15-130 U/L), albumin (ALB) $45.5 \mathrm{~g} / \mathrm{L}$ (normal $35-55 \mathrm{~g} / \mathrm{L}$ ), globulin (GLB) $32.6 \mathrm{~g} / \mathrm{L}$ (normal 20 $-30 \mathrm{~g} / \mathrm{L}$ ), prothrombin time (PT) 11.5 seconds (normal 10 -14 seconds), international normalized ratio (INR) 0.96 (normal 0.8 - 1.2). The HBsAg, anti-HAV, anti-HCV and anti-HEV were negative. Anti-nuclear antibody (ANA), anti-smooth muscle antibody (SMA) and anti-mitochondrial antibody (AMA) were negative. Serum ceruloplasmin (CP) and $\alpha$-fetoprotein were normal. Abdominal ultrasonography was normal. She was admitted to the local hospital and treated with glycyrrhizic acid, for one month, with recovery of liver enzymes levels. However, after this episode, the patient's liver enzymes were frequently abnormal. One year ago, when the patient was admitted to our hospital for the first time, she was alert

Copyright ( 2015, Kowsar Corp. This is an open-access article distributed under the terms of the Creative Commons Attribution-NonCommercial 4.0 International License (http://creativecommons.org/licenses/by-nc/4.0/) which permits copy and redistribute the material just in noncommercial usages, provided the original work is properly cited. 
and oriented to time, place, and person. Her body temperature was $36.5^{\circ} \mathrm{C}$, pulse rate $76 / \mathrm{min}$, respiratory rate 20/min, and blood pressure 120/80 mmHg. Her scleras were icteric. Abdominal examination revealed a soft, although distended and diffusely tender abdomen. There was no rebound tenderness and the bowel sounds were not hyperactive. The liver and spleen were not palpable and shifting dullness was identified. She also presented edema of lower legs. No flapping tremor or palmar erythema were noted. Her liver function tests showed: ALT $335 \mathrm{U} / \mathrm{L}$, AST $657 \mathrm{U} / \mathrm{L}$, TBIL $208.5 \mathrm{umol} / \mathrm{L}$, direct bilirubin (DBIL) $141.3 \mathrm{umol} / \mathrm{L}$, ALB $24.3 \mathrm{~g} / \mathrm{L}$. The laboratory examination findings included: red blood cell count (RBC) 3.04 $\times 10^{12} / \mathrm{L}$, white blood cell count (WBC) $4.3 \times 10^{9} / \mathrm{L}$, platelet count (PLT) $42 \times 10^{9} / \mathrm{L}$, hemoglobin (Hb) $10 \mathrm{~g} / \mathrm{L}$, PT 18 seconds, INR 1.52, negative ANA, negative AMA. Markers of viral hepatitis were negative. Abdominal ultrasonography revealed cirrhosis, which led to a diagnosis of decompensated cirrhosis for this patient. On 5th day of admission, the patient experienced dizziness, headache, nausea and vomiting. A couple of hours later, she reported impaired vision and within several hours, she was totally blind. She could not see movement of hand at the distance of $0.2 \mathrm{~m}$. There was no light perception, although the papillary direct and consensual reflexes to light were normal in both eyes. Ophthalmoscopy and slit lamp examination revealed no abnormalities of the ocular fundus. Ocular movements were normal. A brain CT and transcranial doppler sonography (TCD) showed no abnormality. Electroencephalogram (EEG) tracings were not unusual. Optokinetic nystagmus was absent. Her serum ammonia level was $94 \mathrm{umol} / \mathrm{L}$ (normal range $<33 \mathrm{umol} / \mathrm{L}$ ). The patient remembered eating too much protein diet in the past few days. Therefore, the patient was diagnosed with cortical blindness, secondary to hepatic encephalopathy. She was treated with branched chain amino acid supplements, lactulose, and ornithine aspartate. After 18 hours, serum ammonia level dropped to $21 \mathrm{umol} / \mathrm{L}$, she was alert and oriented, and her vision returned. Because of her elevated liver enzymes and TBIL, the patient was treated with glutathione, silibinin, glycyrrhizic acid, and short course steroid therapy. After 15 days, liver enzymes and bilirubin decreased significantly, and a liver biopsy was performed. Liver histology revealed severe cirrhosis (Figure $1 \mathrm{~A}$ ), and the possible reason was toxin or drug induced liver injury. Her medication history and toxic material contacting history were inquired repeatedly. After 2 days, the patient told us that she had a habit of using dichlorvos painting bed mat to kill bed flea, and she had done this for several years. She told us no other toxins or drugs were used. Therefore, toxic-induced liver damage and cirrhosis were confirmed in this patient. After hospitalization for 44 days, liver function was significant improved. The hepatoprotective drugs and low-dose of steroids were used after discharging from hospital. Eleven months later, the patient admitted to our hospital for the sec- ond time for abnormal liver function. This time, the laboratory results included ALT 304 U/L, AST 292 U/L, TBIL 18 umol/L, DBIL 7.4 umol/L, AKP 123 U/L, GGT 239 U/L, ALB 41.9 g/L, GLB 31.7 g/L, PT 10.1 seconds, prothrombin activity (PTA) 132.6\% (80 - 150), INR 0.94, activated partial thromboplastin time (APTT) 33.2 seconds ( 21 - 38 seconds), fibrinogen $2.04 \mathrm{~g} / \mathrm{L}(2-4 \mathrm{~g} / \mathrm{L})$, thrombin time 19.8 seconds $(14-20$ seconds). The hemoleucogram parameters were WBC $4.3 \times 10^{9} / \mathrm{L}, \mathrm{RBC} 4.68 \times 10^{12} / \mathrm{L}, \mathrm{Hb} 114.4 \mathrm{~g} / \mathrm{L}$, PLT $92.4 \times$ $10^{9} / \mathrm{L}$, ANA H:N 1:1000, IgG $19.3 \mathrm{~g} / \mathrm{L}$ (normal $7.51-15.6 \mathrm{~g} / \mathrm{L}$ ), IgA $2.68 \mathrm{~g} / \mathrm{L}$ (normal $0.82-4.53 \mathrm{~g} / \mathrm{L}$ ), IgM $1.10 \mathrm{~g} / \mathrm{L}$ (normal $0.4-2.74 \mathrm{~g} / \mathrm{L}$ ), C3 $1.12 \mathrm{~g} / \mathrm{L}$ (normal $0.79-1.52 \mathrm{~g} / \mathrm{L}$ ), C4 0.17 $\mathrm{g} / \mathrm{L}$ (normal $0.16-0.38 \mathrm{~g} / \mathrm{L}$ ). Serum ammonia was 22.2 umol/L. Extractable nuclear antigen (ENA) antibody repertoire was negative. Liver biopsy was performed again because of unfavorable therapeutic result and discontinuation of low-dose inhaled corticosteroids, and this time the liver histology showed the portal inflammation, interface hepatitis, cirrhosis and relieved bridging necrosis (Figure $1 \mathrm{~B}$ ). Presence of plasma cells in portal and intra-acinar structures, and rosette formation were features that favored AIH. Iron and copper staining were negative. Six scores were obtained from the simplified criteria for the diagnosis of AIH, the second time in our hospital, therefore supporting a possible diagnosis of $\mathrm{AIH}$. We reviewed the first presentation of the patient, with clinical history and liver biopsy with the same criteria, and the score was 3, and the diagnosis of AIH was excluded. Consequently, the final diagnosis of this patient was toxin-induced AIH. With long term treatment and low dose clycocorticosteroid therapy, the clinical symptoms and liver biochemical indicators of the patient improved.

\section{Discussion}

Dichlorvos is an organophosphoric insecticide, widely used for controlling internal and external parasites in livestock and domestic animal, and also insects in houses and in fields. Dichlorvos is highly toxic by inhalation, dermal absorption and ingestion (9-11). Acute (shortterm) and chronic (long-term) exposures of humans to dichlorvos result in the inhibition of an enzyme, acetylcholinesterase, with neurotoxic effects, including perspiration, vomiting, diarrhea, drowsiness, fatigue, headache, and, at high concentrations, convulsions and coma. Yang J's study aimed to assess the metabolomic profile and related histopathological outcomes of rat plasma after chronic low-dose exposure to dichlorvos, and significant changes in several of the metabolites were found in all the treated groups, compared with the control group $(10,12)$. LysoPC (15:0/0:0), LysoPC (16:0/0:0), LysoPC (17:0/0:0), LysoPC (0:0/18:0), sphingosine, sphinganine, C16 sphinganine, $\mathrm{C} 17$ sphinganine, and arachidonic acid were decreased in the treated groups. LysoPE (16:0/0:0) was increased after dosing with dichlorvos. The livers were damaged following chronic exposure to dichlorvos. 


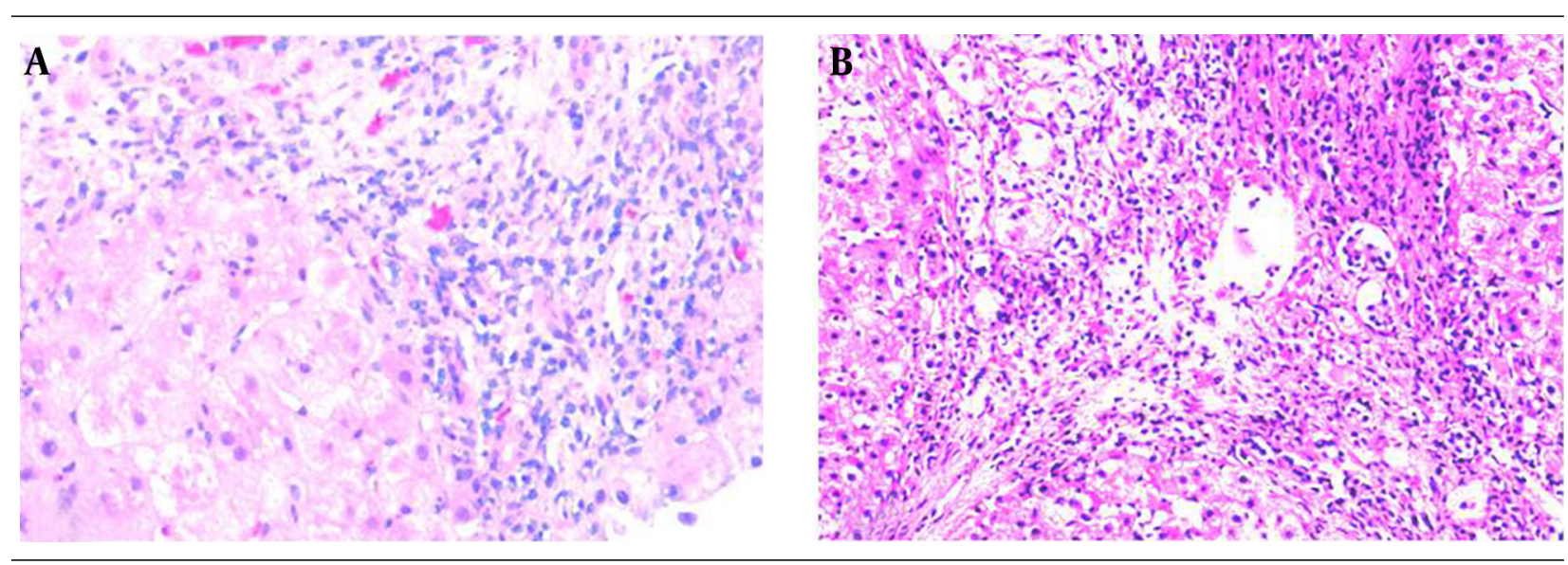

Figure 1. HE staining of the liver biopsy in the first (A) and second time $(B)(\times 200)$

Our patient has a history of long-time low-dose dermal exposure to dichlorvos. Dichlorvos could be absorbed easily through the skin, and a small amount of longterm absorption can lead to toxic-induced liver injury. This patient had abnormal liver function for one and half years, and was admitted four times to hospital without identifying the true reason of liver injury. This rare medical record of long-time and low-dose dermal exposure to dichlorvos provides important clues and basis for the diagnosis of toxic liver injury. Therefore, a thorough medical history from the patient is very important for the physician in the diagnosis of liver diseases (13). Multiple drugs have been linked to $\mathrm{AIH}$ phenotypes. At least three clinical types have been proposed, which refers to DIAILD: AIH with DILI (that is patients with known AIH with high probability of association, which often leads to fibrosis on histology), DIAIH and immune mediated DILI (IM-DILI) with clinical, biochemical, and histological signs similar to $\mathrm{AIH}$. Characteristic for DIAIH are patients with unrecognized $\mathrm{AIH}$ or predisposition to $\mathrm{AIH}$, in whom $\mathrm{AIH}$ is induced by DILI. These cases have a good response to steroids and may present relapse after withdrawal of immunosuppression, with the need for continued immunosuppressive treatment. The chance for an association of drug intake in a patient with first presentation of AIH cannot be ruled out. Drug-induced autoimmune or autoimmune like hepatitis is an acute or chronic, and potentially severe, side effect of drugs (14). Toxin-induced AIH is rare (8). In this patient without history of $\mathrm{AIH}$, the liver enzyme became abnormal with interruption of low-dose corticosteroids, liver fibrosis progressing to cirrhosis. The titer of ANA on the first admittance to our hospital was negative, although on the second admission, the ANA titer was positive. Also, the IgG concentration was higher. The score of simplified criteria for the diagnosis of AIH (15) was 3 on the first admission, and 6 on second admission, and met the established international criteria of AIH. Corticosteroids treatment was effective resulting in improvement of clinical symptoms and liver biochemical indicators. The DILI is thought to involve a complex interaction between chemical properties of the drug, environmental, genetic and pre-existing host factors (16). Recognized predisposing factors include ethnicity, cytochrome polymorphisms, concomitant liver disease, age, nutritional status and diet, gender and pregnancy (16). The DILI is either an overcompensating immune response or a direct cell injury by the drug or its metabolite (17). Toxins can be absorbed and metabolized like drugs. Therefore, we think the mechanism of the toxin-induced liver injury was the same as for a drug, which sometimes persists after drug discontinuation, suggesting that they awaken latent autoimmunity. The release of hepatic antigens and consecutive presentation of these autoantigens by immune cells may lead to a continued autoagressive-immune reaction in genetically susceptible individuals. In a long term follow-up, many of them developed 'true' AIH, with a permanent need for immunosuppression. Therefore, these patients suffer from DI AIH. Cortical blindness is defined as bilateral impairment of vision, with normal ocular structures, normal pupillary light reflex, and absence of nystagmus (18). Hepatic cortical blindness is an unusual clinical complication of hepatic encephalopathy and must be differentiated with nervous system diseases, such as cerebral vascular disease, ophthalmology disorder, or other cause of lead to binocular vision disorder (19). This patient had normal papillary reflexes, and no organic brain lesion on brain CT and TCD, the EEG showed no anomaly, no ophthalmological disorder by ophthalmoscopic exam was evident and slit lamp examination revealed no abnormalities in the ocular fundus (20). This patient had decompensated cirrhosis and impaired vision, which were associated with elevated serum ammonia due to eating too much proteins, and the impaired vision disappeared following cessation of high serum ammonia. Consequently, we considered that the elevated serum ammonia level was the cause of the hepatic cortical blindness in this patient. The prevalence of hepatic cortical blindness is low, and is easy to miss the 
diagnosis. Therefore, in the clinic, when any patient with abnormal liver enzymes and cortical blindness presents, we should think of this disease. Meanwhile, accurate diagnosis is necessary for DILI patients with a history of medication, and several of DILI tend to develop DIAIH. A detailed inquiry of medical history is necessary for the diagnosis of toxin-induced AIH.

\section{Authors' Contributions}

Study concept and drafting of the manuscript: Su Xian Zhao and Li Kong. Recruitment and assessment of patients: Su Xian Zhao, Li Kong. Clinical data collection: Qing Shan Zhang, Yu Guo Zhang, Rong Qi Wang, Yue Min Nan, Ling Bo Kong.

\section{References}

1. Vajro P, Paolella G, Maggiore G, Giordano G. Pediatric celiac disease, cryptogenic hypertransaminasemia, and autoimmune hepatitis. J Pediatr Gastroenterol Nutr. 2013;56(6):663-70.

2. Dehghani SM, Haghighat M, Imanieh MH, Honar N, Negarestani AM, Malekpour A, et al. Autoimmune hepatitis in children: experiences in a tertiary center. Iran J Pediatr. 2013;23(3):302-8.

3. Deutsch M, Emmanuel T, Koskinas J. Autoimmune Hepatitis or Wilson's Disease, a Clinical Dilemma. Hepat Mon. 2013;13(5).

4. Pratico AD, Salafia S, Barone P, La Rosa M, Leonardi S. Type II Autoimmune Hepatitis and Small Duct Sclerosing Cholangitis in a Seven Years Old Child: An Overlap Syndrome? Hepat Mon. 2013;13(12).

5. Rezaee Zavareh MS, Alavian SM, Karimisari H, Shafiei M, Saiedi Hosseini SY. Occult hepatitis $C$ virus infection in patients with autoimmune hepatitis. Hepat Mon. 2014;14(8)

6. deLemos AS, Foureau DM, Jacobs C, Ahrens W, Russo MW, Bonkovsky HL. Drug-induced liver injury with autoimmune features. Semin Liver Dis. 2014;34(2):194-204.

7. Czaja AJ. Drug-induced autoimmune-like hepatitis. Dig Dis Sci.
2011;56(4):958-76.

8. Bjornsson E, Talwalkar J, Treeprasertsuk S, Kamath PS, Takahashi $\mathrm{N}$, Sanderson S, et al. Drug-induced autoimmune hepatitis: clinical characteristics and prognosis. Hepatology. 2010;51(6):2040-8.

9. Ajiboye TO. Redox status of the liver and kidney of 2,2-dichlorovinyl dimethyl phosphate (DDVP) treated rats. Chem Biol Interact. 2010;185(3):202-7.

10. Ogutcu A, Suludere Z, Kalender Y. Dichlorvos-induced hepatotoxicity in rats and the protective effects of vitamins $C$ and E. Environ Toxicol Pharmacol. 2008;26(3):355-61.

11. Binukumar BK, Bal A, Kandimalla R, Sunkaria A, Gill KD. Mitochondrial energy metabolism impairment and liver dysfunction following chronic exposure to dichlorvos. Toxicology. 2010;270(2-3):77-84.

12. Yang J, Wang H, Xu W, Hao D, Du L, Zhao X, et al. Metabolomic analysis of rat plasma following chronic low-dose exposure to dichlorvos. Hum Exp Toxicol. 2013;32(2):196-205.

13. Singh $\mathrm{H}$, Naik AD, Rao R, Petersen LA. Reducing diagnostic errors through effective communication: harnessing the power of in formation technology. J Gen Intern Med. 2008;23(4):489-94.

14. Castiella A, Zapata E, Lucena MI, Andrade RJ. Drug-induced autoimmune liver disease: A diagnostic dilemma of an increasingly reported disease. World J Hepatol. 2014;6(4):160-8.

15. Hennes EM, Zeniya M, Czaja AJ, Pares A, Dalekos GN, Krawitt EL, et al. Simplified criteria for the diagnosis of autoimmune hepatitis. Hepatology. 2008;48(1):169-76.

16. Canbay A, Chen SY, Gieseler RK, Malago M, Karliova M, Gerken G, et al. Overweight patients are more susceptible for acute liver failure. Hepatogastroenterology. 2005;52(65):1516-20.

17. Kaplowitz N. Drug-induced liver injury. Clin Infect Dis. 2004;38 Suppl 2:S44-8.

18. Langer JE, Wilson WG, Raghavan P, Rust RS, Goodkin HP. Extrapontine myelinolysis resulting in transient cortical blindness. Pediatr Neurol. 2010;42(2):154-6.

19. Miyata Y, Motomura S, Tsuji Y, Koga S. Hepatic encephalopathy and reversible cortical blindness. Am J Gastroenterol. 1988;83(7):780-2.

20. van Pesch V, Hernalsteen D, van Rijckevorsel K, Duprez T, Boschi A, Ivanoiu A, et al. Clinical, electrophysiological and brain imaging features during recurrent ictal cortical blindness associated with chronic liver failure. Acta Neurol Belg. 2006;106(4):215-8. 